\title{
Without mapping. \\ Una rilettura di Gn 10,1-32-11,1-9 in prospettiva geoestetica
}

\author{
Guido Boffi
}

The image of the World as a (geographical, philosophical, political, cultural, religious) Whole has influenced and seduced thought, often to the extent of allowing it to lose its sight in what the world itself actually is: recursive connections between elements that lie within one another in dense relationships of interdependency. Not only has the "Whole/ parts" conceptual pair - decisive to geography as well as to philosophical and political ontology - influenced ethnic categorisations, but even/also anthropological and theological evaluations: The Jewish exegesis of the tenth chapter of Genesis provides the prime example of this. Instead, the geo-aesthetic elects the migrating fragment which, expelled from the Whole and refused by the single parts, moves beyond and, from the farthest edge of the new region of the world, claims a place to live, speak, work, and love in sociality.

Guido Boffi is a researcher in philosophy at the Catholic University of Milan. Between 2000 and 2015, he taught Aesthetics, Theoretical Philosophy, History of Philosophy and History of Aesthetics. His fields of interests are German Post-Kantian philosophy (Idealism and Nietzsche in particular), Iconology, Performance Studies, Cultural and Critical Geography, Post-colonial Studies and the intersections between Aesthetics and Politics. He is most recently the author of: Migrazioni. Note di geoestetica I and Per altri atlanti. Note di geoestetica II (Napoli / Salerno 2014).

\section{1.}

Un modello bidimensionale di imago mundi torna con insistenza, lievemente variato, nei testi della tradizione cristiana medievale. È presente ove si voglia rendere il disegno del mondo noto con una sorta di marchio o ideogramma. Si tratta di un'immagine fatta valere come mappa della "terra abitata" ( $\gamma \tilde{\eta}$ oikov $\mu \varepsilon^{\prime} v \eta$, dell'œcumĕne. Molto semplicemente: una circonferenza il cui diametro coincide con la barra orizzontale alla sommità dell'asta di una T inscritta.

Il criterio di lettura è esplicito, ancora lontano dalla logica proiettiva della cartografazione moderna ma non da qualsiasi proiezione. La circonferenza raffigura il globo terracqueo pensato come un disco piatto, il cui orlo estremo è Oceano, che tutto abbraccia e contiene. In esso sfocia senza interruzioni la $\mathrm{T}$ : l'asta è mare magnum, il Mediterraneo; i due bracci della barra superiore indicano 


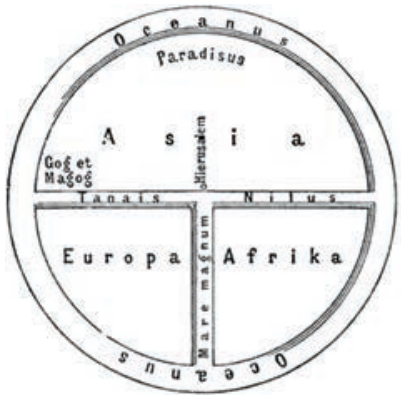

Fig. 1.

invece, nella metà di sinistra, il fiume Don, nella metà di destra il Nilo. Questa T inscritta seziona l'area interna al cerchio in due metà: la superiore rappresenta l'Asia, mentre l'inferiore è partitamente divisa a sua volta in due quadranti, a sinistra Europa, a destra Africa. L'Asia è in parte spazio selvaggio e mostruoso, terra di Gog e Magog. Tuttavia proprio in essa, ai confini della circonferenza, sta il Paradiso Terrestre. Al centro del cerchio-globo: Gerusalemme.

Nel corso del presente articolo vorrei considerare le "avventure" di tale immagine, non ancora conclusesi. Anzitutto intendo mostrare il nesso intrinseco che lega questo modello di mondo con la mappa mentale implicita in Gen 10,1-32. Secondariamente cercherò di esibire le potenzialità che questo breve capitolo dell'Antico Testamento custodisce sotto l'epidermide così asciutta della sua redazione. Dopo essermi occupato, nel terzo paragrafo, della differenza tra gli antichi modi di rappresentazione "corografica" e "geografica" circa il disegno del mondo come Tutto, nel quarto vorrei mostrare in che modo l'antica esegesi giudaica operi un'interpretazione di Gen. 10 dal pesante carico etno-antropologico, oltre che teologico, influente pure sulla cosmografia e la corrispondente cartografia. Nel quinto paragrafo cerco di mettere a frutto una recente ripresa artistica di tale immagine e la poetica di un'opera che ha messo al centro l'idea di mondo quale totalità aperta. Mi concentrerò sulla prospettiva geoestetica per mostrare come possa servire a scardinare i pericolosi dispositivi totalitari di territorializzazione e identitarizzazione, e perciò a immaginare di nuovo il mondo. ${ }^{1}$

1 Ho abbozzato il costrutto di una "geoestetica" in Boffi 2014a, in particolare cfr. pp. 25-35; Boffi 2014b. Da un punto di vista complementare, il progetto condotto dal geografo Mario Neve, del quale cfr. almeno Neve 2004, pp. 1-21; Neve 2005, pp. 153-170; Neve 2008, pp. 165-176. Inoltre si veda il volume con importanti contributi di autori vari Quirós / Imhoff 2014. 


\section{1.}

Ora, prima di ulteriori osservazioni, mi sembra significativo iniziare dall' annosa questione stilizzata nella mappa di fig. 1. Duplice questione perché geografica e cosmologica ad un tempo. Infatti, nella sua architettura semantica tale semplicissimo il diagramma contiene una dissonanza teorica di lunga data e densa di riverberi, di eco lontanissime. Per un verso, la rappresentazione piatta, il disco della terra-ecumene, e insieme, d'altro verso, la sua rappresentazione sferica come corpo celeste. Non è qui il luogo anche solo per una veloce ripresa o sintesi della storia concettuale di queste idee. Tuttavia è opportuno riaprire per un istante l'intervallo di senso da cui si produce la discordanza delle raffigurazioni e dei loro significati.

Basti ricordare essenzialmente che la Terra in quanto sfera celeste compare fin dai disegni dell'astronomia greca (si pensi già ad Aristotele per esempio, o a Cratete di Mallo, filosofo stoico del II secolo a.C. che vi si dedicò con attenzione specifica), mentre l'immaginazione della Terra quale distesa piatta appartiene al racconto biblico. Due immagini opposte, dissonanti appunto. Eppure il diagramma di fig. 1, cosiddetto "modello T-O", tenta la loro conciliazione. Non una qualsiasi, si badi, ma precisamente quella "verificata" dal segno cristiano. La raffigurazione pseudo-cartografica delle parti della terra, "corografica" come si era soliti dire per indicare l'approccio differenziale ai luoghi, ${ }^{2}$ è sottoposta alla visione geografica che si prefigge di cogliere invece la superficie terrestre nella sua unità e interezza relazionale, ovvero come mondo. Nella nostra accezione d'uso, il termine "geografia", lungi dal ridursi a descrizione della terra, è sempre sapere di spazi umanizzati, cioè di relazioni e politiche negli/degli spazi. Ma, attenzione, nel caso dell'enciclopedia del sapere che produce il "modello T-O" caratteristico del Medioevo cristiano, si tratta di geografia religiosa. La totalità rappresentata viene cioè messa in asse con il symbolon del Dio che in Cristo ha posto la sua tenda sulla terra e fra le tende degli uomini. È "soggetta" alla visione del Tutto in quanto creato, e per ciò all'idea della perenne mobilitazione pneumatica dell'Uno nel Tutto, dello hen che attira e unifica al proprio centro il pan, in vista dell'intera ricapitolazione senza resti di quanto vive in cielo e in terra, panta en pasin (1 Cor 15,28).

Quasi ogni mappa cristiano-medievale illustra non soltanto l'intero e le parti, l'unità e il centro, ma anche gli inizi. Come abbiamo visto in fig. 1, alla sommità della mappa è posto il Paradiso. Sta ai confini del mondo, con valenza di giardino dell'Eden ma infine pure di destinazione ultima. Non v'è qui, dunque, rappresentazione soltanto spaziale della terra nella sua problematica congiunzione di bidimensionalità piatta e sfericità del globo celeste, e neppure al modo che si attesterà a partire dalla cartografia moderna. Anzi, a ben vedere è in gioco una

2 Si tratta di un'approssimazione non così stringente rispetto all'uso originario di chorographia, su cui torno tra breve. 
figurazione soprattutto temporale. O meglio, rappresentazione della vetta dalla quale la mentalità dominante dell'epoca vede "discendere", "decadere" il tempo e dove esso dovrà fare ritorno come alla sua patria ultraterrena. Passato presente futuro "in uno" con lo spazio del mondo abitato, dell'ecumene. In definitiva, Atene $e$ Gerusalemme? In un certo senso sì. In verità però, ho già detto, il segno cristiano domina il modello dell'improbabile conciliazione. Siamo di fronte piuttosto a una ritemporalizzazione-sacralizzazione dello spazio abitato, a un'iscrizione "corografica" di Atene nella geografia totale della Gerusalemme celeste. Questione decisiva, certo, e di primaria importanza per la tradizione della cartografia non soltanto religiosa. E tuttavia non è ciò che ora m'importa di più. ${ }^{3}$ M'interessa mantenere l'attenzione sul modello T-O, nella consapevolezza del problema cui dà forma e del quale schizza una conciliazione in figura.

\section{2.}

Nel 1472, vent'anni prima che Colombo calchi piede sulle attuali isole Bahamas dando avvio alla colonizzazione delle Americhe assai più che alla loro "scoperta", in uno dei centri commerciali, economici ed ecclesiastici della vecchia Europa, Augsburg, vengono pubblicate le celebri Ethymologiae (red. 623) di Isidoro di Siviglia. Il volume è impreziosito da pregevoli incisioni, fra cui quella con cui Günther Zainer riproduce l'imago mundi di cui stiamo trattando. Solo che si apra l'opera al Libro quattordicesimo, "de terra et partibus", ed ecco il cerchio dell'Oceano che avvolge il disco del mondo.

Ecco la T inscritta nella $\mathrm{O}$ (fig. 2).

Ora zumiamo dalla pagina all'incisione (fig. 3). Confrontiamola con lo schema di fig. 1, quali novità? Osservando più da vicino, riscontreremo elementi aggiunti ed elementi tolti. Due e due.

Iniziamo dai primi, dai nuovi elementi introdotti, che balzano agli occhi in modo più immediato.

a) Anzitutto i termini esterni alla circonferenza, i nomi delle direzioni cardinali lungo le quali è possibile percorrere la superficie terrestre. A partire dall'alto in senso orario: oriens, meridies, occidens, septentrio. Ci sono tutti. Eppure non possono che sembrarci fuori posto. Infatti, alla sommità della fig. 3 è "escritto" oriente, ciò che costituisce precisamente un "disorientamento", un disassamento simbolico e una rotazione a 90 gradi in senso antiorario dell'assetto cartografico che siamo abituati a leggere e concepire. Ai nostri occhi tutta la mappa risulta ruotata. Mentre alla sommità per noi si colloca settentrione, qui sta oriente. Prescindendo dal fatto che tale configurazione così anormale ai nostri occhi

3 Allo stesso modo qui non m'interessa trattare la rottura moderna di questa stessa modellizzazione, la soluzione di continuità sopravvenuta per spinta delle imprese dei navigatori del Quattro-Cinquecento. 


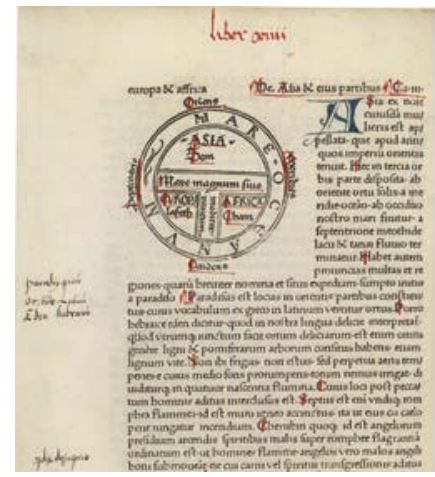

Fig. 2.

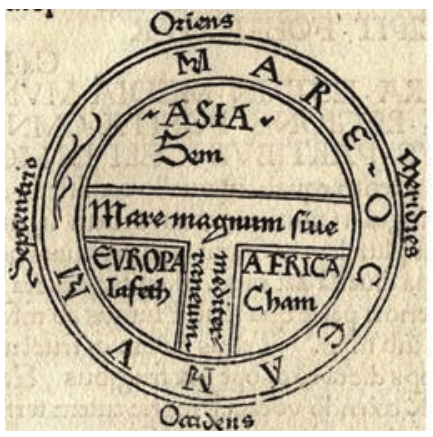

Fig. 3.

corrisponda invece a una normalissima consuetudine dell'epoca, presumo che fig. 3 altro non faccia se non esplicitare il significato muto di fig. 1 . Ne è prova il permanere della medesima ripartizione e collocazione delle terre (i nostri "continenti") rispetto all'asta della T, sorta di axis mundi cartografico. Anche nella versione di fig. 1, l'Asia ricopre interamente da sola la sezione superiore. Dunque, oriente era immaginato in cima già in precedenza, anche se non indicato per iscritto. Perché? Proprio per la matrice religiosa dell'impianto: impianto: in Asia si diceva che si trovasse l'originario Paradiso Terrestre. In quanto simbolo spirituale, oriente sta in alto e illumina il tutto: ex oriente lux; solem orientem. ${ }^{4}$

Prima di proseguire, va fatta un'ulteriore valutazione in merito. Si deve tenere presente che anche un modello così semplificato era disegnato per svolgere le sue ordinarie funzioni pedagogica e, come la chiameremmo oggi, performativa. Non

4 Non può non venire alla mente, per controcanto, l'impresa che Edward Wadie Said ha dedicato alla demistificazione di tutto ciò che è disceso dal "mito" di oriente in quanto relazione di potere-sapere che l'Europa ha instaurato nei suoi confronti. Si veda almeno il celebre saggio Said 1978. 
intendeva affatto delineare-dimostrare dove fossero ubicati separatamente i particolari continenti della terra. Piuttosto, all'interno di un sistema simbolico qual è quello che detta la geografia di questa imago mundi, istruiva il criterio spirituale in base al quale essa avrebbe dovuto insegnare a leggere (religiosamente) la geografia del mondo-tutto. Era funzionale a territorializzarne e ad abitarne gli spazi. In definitiva, serviva a disegnare i confini che avrebbero prodotto le cosiddette identità culturali. Doveva far credere che queste esistano per natura proprio così come sono, al pari di un monte, un fiume, un bosco. In tal senso, del resto, non si devono leggere sempre anche le direzioni cardinali? Soltanto il torpore dell'ovvietà può frenare o bloccare la consapevolezza del fatto che si tratti di emisferi culturali. Oriente, meridione, occidente e settentrione non esistono in natura. Sono qualcosa che c'è in quanto inventato. Sospettose guardie di confine di un progetto politico. Con ogni probabilità il medesimo progetto delle identità culturali, battezzato appunto "Occidente". Sono fatti storici, geografici (cioè politici), culturali. Li ha prodotti l'uomo per i propri interessi entro pratiche di potere ogni volta localizzate (accreditate come il sapere "scientifico", "esatto", "completo", "utile"!). Ciò non deve affatto stupire. Il mapping - oggi i geografi chiamano così la cartografazione - è valso a lungo in quanto unico strumento tramite il quale chiunque avrebbe potuto (ma anche dovuto) farsi un'idea della terra e delle sue località più o meno estreme, selvagge, barbare. In altri termini, era utilizzato per produrre una determinata realtà dagli effetti della credenza nella sua rappresentazione. In nessuna maniera ha mai costituito il dispositivo grafico di un ricalco, una pura registrazione di dati. ${ }^{5}$ La rappresentazione simbolica valeva ben più di quella empirica: la credenza doveva alimentare la forza della tradizione. In modo evidente, l'elaborazione, la valorizzazione, il progresso della cosiddetta cultura occidentale ne sono dipesi per vincoli decisivi, ben poche volte pacifici. ${ }^{6}$

\section{3.}

b) Veniamo al secondo elemento di novità esibito da fig. 3. Il focolaio teorico di questo mio articolo vi trova buone ragioni per ravvivarsi. Nelle aree interne sezionate dalla $\mathrm{T}$, accoppiati rispettivamente all'indicazione delle tre grandi partizioni "continentali", leggiamo i nomi dei figli di Noè. Asia-Sem, AfricaCham, Europa-Jafeth. Di qui, dai figli dei figli di Noè e dalla loro distribuzione per la terra, prende corpo la cosiddetta "tavola dei popoli". Dunque, fig. 3 riproduce Gen 10, che nei suoi aridi elenchi trasmette una concezione genetica dell'abita-

\section{Cfr. Crampton 2010.}

6 Su tutta questa problematica si veda senz'altro il rilevante volume pubblicato di recente da M. Neve 2016, in particolare pp. 7-26, 189-317. Inoltre almeno il precedente lavoro di Jacob 1992; e il fondamentale testo di Farinelli 2009. 
zione del mondo, cioè di come la terra diventi ecumene. Ma, attenzione, ri-produce anche nel senso che lo produce di nuovo introducendo delle tre grandi partizioni della superficie terrestre, mentre il testo masoretico si limita a esporre le discendenze. Si tratta di un'aggiunta per nulla trascurabile, che fa supporre il filtro dell'esegesi giudaica antica, segnatamente del libro dei Giubilei, ${ }^{7}$ così importante nella ricezione di tale capitolo veterotestamentario - e su ciò tornerò nell'ultimo paragrafo.

Vale la pena di iniziare a dire qualcosa circa questo capitoletto del Genesi. Con ogni probabilità l'ascolto che siamo abituati a prestargli è istruito da lineamenti di filosofia e/o teologia della storia. È vero, può essere recepito anche così. Fuor di dubbio, però, esso delinea implicitamente una propria geografia ed è su questo piano di consistenza che vorrei saggiare via via posizione e determinazione del problema filosofico che esso dà a pensare. Delinea una geografia mentale in buona parte forse anche immaginaria, ma alimentatasi di precise nozioni provenienti dal mondo commerciale fenicio, da una cultura che sta alle sue spalle sin dai secoli VIII e VII. ${ }^{8}$

L'analisi critico-letteraria di Gen 10 ha ormai stabilito che il testo ebraico possiede la forma attuale provenendo dall'interazione tra le sue componenti redazionali sacerdotale $(\mathrm{P})$ e jahvista $(\mathrm{J})$. Va ricordato che modifiche e ampliamenti sono intervenuti a dettarne l'evoluzione diacronica corrispondentemente ai mutamenti del suo contesto genetico. In pratica si è assecondata l'esigenza di aderire alle diverse situazioni storico-politiche determinatesi nel territorio siropalestinese soggetto al dominio, prima, neo-babilonese e, poi, persiano. Quanto al sapere geografico implicito in questo capitoletto, oggi risulta comunque molto difficile stabilire con esattezza i limiti delle competenze dei suoi diversi redattori. Di conseguenza diventa arduo fissare fin dove il lettore che si senta stimolato a identificare direttrici e luoghi di destinazione possa o debba integrarlo facendo intervenire proprie nozioni.

Ebbene, se in generale la cartografazione si è svolta secondo quanto ho provato a dire più sopra, in tale cornice occorre disporre anche Gen 10. Possiamo ipotizzare che per i suoi primi lettori e uditori il racconto funzionasse come un modo (o anche: il modo) per farsi un'idea di un vasto spazio geografico altrimenti puramente fantasticabile. La mappa che vi era ricavabile non registrava territorialità già stabilite. In tal senso Gen 10 , mentre parla dell'orizzontalità della terra sulla quale s'inaugurano le linee divergenti delle tre genealogie, svolge un'esemplare funzione performativa di una concezione del mondo, cioè di una complessità di relazioni. Non si limita a elencare e ridurre in ordinamenti ge-

7 Il commento a Gen 10,1-32 redatto nel libro dei Giubilei (8-10) è accessibile in versione italiana con apparato filologico nel volume Sacchi (a cura di) 1981, vol. I, pp. 258-270.

8 Cfr. Prato 1999, pp. 511-546, ora anche in Prato 2010, pp. 88-124, in particolare, pp. 108-111; Prato 2013, pp. 135-160, in particolare pp. 136-139. 
nealogici i processi primitivi della distribuzione e dell'abitazione della terra da parte dei gruppi umani discendenti da Noè («nazioni» per il testo, che necessariamente non può avvalersi di tale termine se non in un'accezione ancora esclusivamente linguistico-culturale). Certo parla di moltitudini e di unità, di molti gruppi per molti luoghi a partire da un'unità-centro. Proprio così facendo, tuttavia, produce una rappresentazione finalizzata a un'identità portante di ciò che oggi siamo soliti chiamare, talvolta con superficialità, "cultura occidentale" o "europea" ma anche "cultura giudaico-cristiana". Lo fa istruendo in un preciso modo la memoria culturale.

\section{4 .}

c) Ora, e più brevemente, gli elementi tolti da fig. 3 rispetto a fig. 1. In primis risulta cancellato il decisivo fattore di temporalizzazione. Mancano, in sostanza, gli inizi e la cosa ultima, gli éschata della mappa. Non compare più l'indicazione del sommo bene. È cancellato il Paradiso iscritto ai confini del mondo e della temporalità storica. Fig. 1 risultava del tutto orientata sull'Eden, non a caso fatto coincidere con i limiti estremi sul punto cardinale di oriente. Cartografia della trascendenza? Sia come sia, la caduta dei confini estremi del tempo marca una differenza dalle conseguenze non trascurabili. Come se l'inizio del tempo venisse tradotto-tradito nello spazio - ciò che poi effettivamente accadde con l'ingresso nella modernità. Può sembrare una quasi totale mondanizzazione dell'impianto concettuale, pur restando fermo il diretto riferimento alla Scrittura con l'introduzione "stenografica" di Gn 10.

Forse proprio perché si è sempre concepito che gli uomini debbano guadagnarsi su questa terra l'accesso a quell'Aldilà che resta ogni volta tale, appunto per ciò una maggiore consapevolezza della distanza profana può comportare la completa, perfetta fiducia nello stato supremo e definitivo, neppure più da rintracciare per segni bensì soltanto da presupporre, da postulare, da credere. In altri termini, ne può conseguire che il segno del Paradiso schizzi via oltre qualsiasi contorno di mappa, oltrepassando all'infinito ogni confine e qualsivoglia cartografia, in una pura extratemporalità ed extraterritorialità. Non è diagrammabile, perché le mappe servono a innescare processi di territorializzazione e costituzione di luoghi. Rendono sicuro il transito, fissano i confini che producono le identità. Di tutto ciò il Paradiso invece rappresenta puramente e semplicemente l'oltreconfine. Un oltreconfine spostato - "rimosso" assai più che annullato.

d) Infine il secondo elemento scomparso, il centro della circonferenza: Gerusalemme. Analogamente a quanto ho scritto a proposito di a), sono portato a ritenere che anche la sua concezione resti fungente e che il nome taciuto del centro sia in verità implicito. Precisamente il richiamo in parallelo a Gen 10 corrobora tale mia convinzione. Il racconto di questo capitoletto della Scrittura 
insiste nel parlare di «nazioni disperse» (v. 5), ovvero di «famiglie» e «nazioni» che «si dispersero» (vv. 18; 32) sulla terra dopo il diluvio. Ora, "disperdere" non significa frammentare né semplicemente perdere. Anzi, esprime tutt'altro. Dice bensì di un disseminare e un dividere, anche nelle forme riflessive dei verbi, ma insieme di un accadere o mandare secondo direzioni, per quanto varie queste possano capitare e aprirsi qua e là. Tuttavia, in generale non v'è "movimento verso" se non ogni volta sulla base di un moto da luogo. Gen 10, proprio mentre non nomina affatto Gerusalemme, adotta implicitamente tanto lo schema centro/ periferia quanto quello dei rapporti di vicinanza/lontananza: ma da dove? L'ovvia risposta non può che essere: dall'Israele antico, ombelico del mondo.

Torniamo adesso alla mappa. A proposito di fig. 1, ho scritto: non corografia bensì geografia religiosa. Ebbene, la corrispettiva fissazione dei nomi dei tre figli di Noè, nell'appaiamento agli spazi continentali, vale per determinare un inizio che apre durate alla luce del Testo sacro. Gen 10 funge pertanto da presupposto anche per questa variazione del modello T-O. Al pari del puro divergere delle nazioni disperse profilato nel testo, la divisione dei continenti sulla mappa rinvia genealogicamente a Israele, al centro-unità Gerusalemme. Pur in assenza di una sua collocazione in una prassi cartografica consolidata al modo di quella greca antica, Gerusalemme, ancorché inespresso, permane il luogo da cui muovono i percorsi diffusivi dell'abitazione umana dopo il diluvio universale. Funge da tacito centro ideale che universalizza e ritemporalizza religiosamente la geografia - non (ancora) invece come un'entità storico-religiosa che territorializzi gli spazi.

\section{2.}

Gen 10 inventaria le genealogie dei discendenti dei tre figli di Noè. Anche qui, su di un piano che forse potrebbe essere detto di geografia antropica, siamo indotti a riconoscere un rapporto di derivazione dei molti (delle moltitudini) dall'uno. Gen 10,1-32 elenca persone, famiglie, tribù, tutte discendenti dal patriarca Noè. Però, si badi, li nomina raggruppandoli sempre soltanto in quanto «si dispersero» tra isole, montagne, paesi e città. Per dir così, ne geolocalizza l'andare. Parla di movimenti di generazioni e generazioni di genti, non di queste o della loro quantità. Racconta, in tal senso, di migranti. Schizza continuamente direzioni, spostamenti, linee di confine. A tale proposito, la stessa consueta denominazione "tavola dei popoli" suona falsa. Errata, in definitiva. Non tanto di popoli radunati (tanto meno "radunati nell'Uno-Stato", evidentemente), di nazioni costituite. Si tratta piuttosto di dispersioni di moltitudini povere e di popolamenti - e la differenza è sostanziale.

Quel breve capitolo del Genesi inquadra la potenza di dislocazione e divergenza. D'altro lato, però, è la stessa potenza costituente luoghi e soggetti collettivi. I movimenti trasmettono dunque un ritmo, quello di una potenza genetica di slegare e collegare, di venire e andare, di dividere e riunire. L'aridità degli 
elenchi genealogici di Gen 10, la neutralità del prospetto, la facie tabulare e compilatoria racchiudono in realtà i "divenire" affermativi, espansivi, moltitudinari di geografie esistenziali e immaginarie: ciò mi pare l'elemento decisivo dell'interpretazione. La superficie, per dir così, statistico-quantitativa del testo esplode, quando l'innesco del suo significato venga acceso dalla potenza moltitudinaria di movimento-popolamento che instaura congiunture viventi e abitazioni, che apre cerchie d'essere.

\section{1.}

Facciamo attenzione un istante al tenore epistemico del discorso. Il testo non pone a oggetto il popolo (più popoli), in quanto proprio il suo presunto "oggetto" non è, in verità, tale. Vi sono processi, divenire, movimenti e azioni assai più che oggetti dati, statici - così come vi sono diversi processi di soggettivazione nella funzioneautore svolta da ripetitori orali e redattori del testo (gli uni e gli altri, vari involucri del suo "quasi-soggetto"). Appunto, divergenze dispersive di popolamento, divenire-soggetto collettivo di famiglie, tribù, moltitudini più o meno estese anziché popoli e nazioni già fissati. Processi e movimenti che vanno a realizzare variamente un'ecumene-mondo ancora tutta da farsi, nella sua bellezza inaugurale.

Se così stanno le cose, il testo svolge una funzione performativa, al pari della mappa - come ho cercato di chiarire prima in un'osservazione che qui torna circolarmente su se stessa per completarsi. Chiamiamola funzione-mondo. Gen 10 scontorna ora indirettamente ora espressamente territori e suoli, isole e montagne, paesi e confini naturali. Insomma, parla della superficie terrestre che da sempre la geografia descrive e le carte rappresentano parzialmente. Racconta di quella terra che la filosofia pensa nel suo consistere in insiemi di rapporti tra molteplicità (mi rifaccio soprattutto alla linea: Kant-Nietzsche-Deleuze).

Ora, per quanto ciò sembri restituire il "fatto", non basta invece a penetrare la superficie del testo a proposito del "come". Occorre di nuovo trattare dei movimenti dispersivi, delle divergenze originarie che s'inscrivono sull'epidermide della terra. Gen 10,1-32 mostra come la superficie terrestre divenga ecumene per la potenza genetica del diffondersi, dell'emigrare. Per tale potenza essa si spartisce in nuove regioni del mondo abitabili dalla discendenza dei figli di Noè. Gen 10,132 intende illustrare, e proprio sfruttando il contrasto di una scrittura il più asciutta possibile, in quale modo la terra si trasformi in una complessità di relazioni e convivenze, analogamente a come fig. 3 fotografa questo stesso processo genetico di abitazione-formazione del mondo, rispetto a fig. 1, inserendo i nomi dei figli di Noè al di sotto dell'indicazione dei continenti. Mentre descrive geograficamente luoghi in via di costituzione, e che soltanto molto dopo verranno territorializzati da popoli-nazioni, Gen 10,1-32 fa mondo. Lo produce-esegue esperienzialmente come pure, almeno in parte, in modi materiali. Esperienzialmente, nel senso che diviene pensiero, rappresentazione, affetto di una potenza e di un'enunciazione 
corali, collettive, essendo testo sacro letto, riletto e commentato da generazioni di generazioni di discendenti del patriarca. È esperienza genetica di una soggettività sociale affermativa, cooperativa, apertasi nella pluralizzazione e correlatività generali che costituiscono l'ecumene. Ma Gen 10 produce anche in modi materiali il mondo in quanto il racconto si fa respiro, saliva, labbra, voce che esce, gesto, luogo d'incontro, abbraccio, mani che si stringono, passi che incedono, essere comune dei molti o dei pochi che della terra fanno territori strappati, abitati.

Mi pare che così, e in molte altre maniere ora non ripercorribili, il testo esegua la sua funzione-mondo, sottraendo la sua superficie fredda a una lettura piattamente protocollare, mortificante. Gen 10,1-32 non detta il referto di un esame autoptico. Risillaba relazioni che stanno sempre l'una nell'altra mentre apronoestendono cerchie di essere comune. L'insieme in divenire di tali relazioni mobili corrisponde a ciò che, in accezione non descrittiva, ho chiamato "processo", polo quasi-oggettivo. Dunque, se così stanno le cose, il testo fa mondo analogamente a come il mondo fa testo (rifà, ricrea): un tornare su di sé e un rilanciare in senso spiraliforme, performativo, non una circolarità chiusa. Ciò costituisce già un primo chiarimento sulla natura di Gen 10. Una prima indicazione per una lettura che non lo richiuda sui suoi elenchi bensì lo apra a geografie di relazioni complesse. Ma forse occorre altresì un chiarimento sul pensiero del testo. In verità non siamo noi a pensarlo, mentre è piuttosto esso stesso a imporre una questione necessaria, che si trasmette esemplarmente per noi, alle relazioni del nostro presente. Vale a dire che si trasmette nei vari "mondi" i quali oggi producono, spesso frammentariamente rispetto alle dinamiche di globalizzazione, il mondo. Qui, mi pare, possiamo riconoscere il piano di consistenza problematica composto unitariamente da testo e figure-mappe.

\section{2.}

Non ho usato a caso il termine "migranti", a proposito delle generazioni di genti che il testo di Genesi fotografa nel transito costituente. Tentando l'archeologia del concetto europeo di migrazione, m'è capitato non molto tempo fa di occuparmi appunto di Gen $10 .{ }^{9}$ Non che questo capitoletto dell'Antico Testamento tratti in modo diretto di migrazioni, lo sappiamo bene. Tuttavia la grande dispersione delle "nazioni" sulla faccia della terra possiede i tratti di un innumere migrare senza di cui non v'è produzione dell'ecumene. Ora, tra i gruppi umani migranti che provengono dai figli dei figli di Noè v'è quello delle genti dette "semitiche": letteralmente, coloro che appartengono alla stirpe di Sem, con discendenza sino ad Abramo (secondo il passo di poco successivo: Gen 11,26). In questo specifico caso, può apparire ancor meno implausibile l'appartenenza costitutiva proprio dell'emigrare alla storia culturale dei semiti, solo che si riesca ad ascoltare la

9 Cfr. Boffi 2014a, pp. 105-115, in particolare pp. 105-107. 
radice del termine stesso "ebreo" (che significa: "passare", "attraversare", "oltrepassare") e a recepire il significato di "migratore, emigrante" nel vocabolo perátes del testo greco (LXX), che rende l'antico lemma ebraico. ${ }^{10}$ Dunque, se così stanno le cose, possiamo provare a intendere in quale senso Gen 10 apparecchi simultaneamente una sorta di "tavola delle migrazioni" in quanto "tavola dei popolamenti". L'immagine del popolo ebraico come errante-migrante vale anche come una formidabile sineddoche che non radica affatto in senso etnico ma che apre al senso inesauribile di movimenti e dispersioni che fanno il mondo-tutto.

Precisamente tale significato ulteriore permette ora uno snodo argomentativo. Esso si squaderna non appena alla lettura del decimo capitoletto si faccia seguire quella della successiva pericope della torre di Babele (Gn 11,1-9). Notoriamente, questo episodio del Genesi si conclude di nuovo con la dispersione geografica della moltitudine di nazioni e, appunto, l'antonomastica Babel linguarum circa la quale molto, persino troppo, s'è fantasticato e giudicato. S'è immaginato, pensato, scritto, dipinto di tutto e di più, facendo proliferare un immaginario collettivo incentrato soltanto sull'asse di una travisata, "cattiva" verticalità. S'è intesa la tracotanza umana e il risentimento (davvero "umano, troppo umano") di Dio, che la punisce umiliando e confondendo. Tuttavia, com'è stato osservato con finezza, occorre mantenere l'attenzione al contesto che fornisce il senso complessivo. ${ }^{11} \mathrm{~A}$ evitare gravi fraintendimenti, occorre tenere presente che anche questo episodio s'inserisce nella sezione (1-11) del libro veterotestamentario destinato a narrare la creazione del mondo. In quanto racconto delle origini, espone i modi e gli effetti di una genesi. Però qui la posta in gioco, più ancora che la creazione dell'imprecisata terra, è la formazione del mondo abitato nella pluralità storico-materiale di linguaggi, culture, civiltà. In tal senso è opportuno chiedersi se Gn 11,1-9 non fornisca piuttosto una rappresentazione genetica della «realtà attuale del mondo, che non è unitaria né univoca», esprimendo con l'episodio di Babele «semplicemente il passaggio dal semplice al complesso» ${ }^{12}$.

\section{3.}

Facciamo il punto dell'interpretazione. Chiarito in sede critico-esegetica, fra l'altro, che la famigerata torre è un parto ideale della versione greca dei LXX - poi ripresa nel latino della Volgata -, mentre l'antico testo ebraico parla piuttosto dell'edificazione di una città e di una struttura fortificata al suo interno, una sorta di cittadella o di rocca; chiarito che la verticalità espressa dal brano va anche - se

10 Filone d'Alessandria fu tra i primi a commentarlo, anche se in una proiezione interamente ultraterrena: cfr. Filone d'Alessandria, De Migratione Abrahami, 20. Me ne sono occupato in Boffi 2014a, pp. 73-84, in particolare p. 77.

11 Cfr. Prato 2008, pp. 53-62, ora anche in Prato 2010, pp. 76-87.

12 Ivi, p. 77. 
non soprattutto - nella direzione di una discesa di Dio, il quale constata l'unità di linguaggio degli umani e la vuole disperdere-diffondere - mentre a ben vedere non condanna la realizzazione non impossibile del loro progetto cooperativo; ciò detto, allora sembra plausibile concludere che il significato di questo celeberrimo episodio narrato dal capitolo 11 custodisce esso stesso le potenzialità genetiche dell'ecumene. Racconta il popolamento del mondo quale pluralizzazione e divergenze di molteplicità. Mostra come inizino a organizzarsi e a organizzarlo socialmente differenti civiltà e culture. L'intervento divino trasforma una originaria unità indifferenziata, come tale inadatta alla sopravvivenza dell'umanità, in una variegata molteplicità storica. Apre a un pluralismo bensì impegnativo ma vitale.

Dunque, interpretando l'agire divino e la conseguente mescolanza delle lingue come punizioni di una colpa, s'è trascurata quasi sempre una caratteristica nota geografica, essenziale tanto al racconto quanto, di necessità, alla sua comprensione. La si può cogliere passando dal piano di lettura dell'ascensionalitàverticalità tracotanti a quello dell'orizzontalità costituente, cioè dei disegni/reticoli di relazioni, concatenamenti, connessioni. Il che è come dire: il piano di consistenza e di mobilitazione generale del mondo - ma si potrebbe anche dire al plurale: dei diversi mondi dentro al divenire-mondo. Perché il punto di vista geografico altro non assume se non il ricorrere di trame spaziali di cose, il multiverso di velocità intrecci frazionamenti, di forme di socialità e rapporti di potere, insomma quei ricorsivi insiemi relazionali nei quali sono presi i viventi (non soltanto umani) per il fatto stesso di essere localmente e universalmente vivi.

Giunto a questo punto, mi sembra di poter concludere una seconda osservazione. La rottura del regime monolinguistico e l'esplosione del plurilinguismo presentati da Gn 11,1-9 corroborano e amplificano il significato già espresso dai movimenti contrapposti del convergere in uno spazio e del successivo disperdersi lungo tutta la superficie terrestre. Ovvero, il significato contenuto in Gen 10,1-32 e nella corrispondente fig. 3: una genesi relazionale della complessità dall'unità semplice. La creazione dei molti dall'uno.

Ebbene, occorre considerare proprio questo passaggio genetico in modo più stringente per noi, cioè all'altezza della nostra realtà. Ma prima di arrivarvi vorrei provare a entrare in modo più perspicuo nel taglio apertosi sul piano geografico ancor prima che geofilosofico.

\section{3.}

Anzitutto rileva tornare all'antica distinzione tra geografia e corografia. Vi si può vedere formulata per la prima volta in concetti geografici la questione uno/molti, alla quale è riconducibile quella specificatasi poi come rapporto tra intero e parti. Mi pare che da tali nodi teorici provengano gli interrogativi circa le attuali dia- 
lettiche locale/globale e regione/mondo, cui infine vorrei giungere per via argomentativa. Conviene procedervi per gradi.

Com'è stato osservato, la questione uno/molti già colta nell'età antica è stata costantemente riproposta dalla cartografia in tutti gli sviluppi - e non a caso. Ma, ben più a fondo, essa è rimasta alla radice e nella linfa del pensiero geografico toutcourt. ${ }^{13}$ Almeno dal mio punto di vista, non v'è salto di conoscenza del pensiero geografico che non ne abbia implicato una simultanea riproposizione e un diverso sviluppo. Non tuttavia al modo di una fra le tante e diverse, bensì precisamente come la questione della geografia. Costituisce la messa in discussione geografica e geofilosofica per eccellenza. Lo è in quanto ricerca-invenzione di totalità, di insiemi, ovvero disegno dei rapporti e delle connessioni tra unità e molteplicità, tra intero-tutto e parti, tra mondo e regioni.

\section{1.}

Gli antichi ebbero una prima consapevolezza di tale problematica. Per convincersene basta lasciare la parola un istante a Strabone, il secondo, dopo Eratostene, tra i pionieri del pensiero geografico - predecessore, a sua volta, del terzo padre fondatore, Tolomeo. Storico e geografo contemporaneo di Augusto, Strabone stese in età già matura i suoi Geographika, aggiornando in senso critico la prima opera così intitolata all'incirca un paio di secoli prima proprio da Eratostene. L'avvio è esplicito:

La scienza geografica [...] rientra anch'essa a buon diritto fra le occupazioni del filosofo [...]. I primi infatti a occuparsi di geografia furono Omero, Anassimandro di Mileto ed Ecateo [...]; quindi Democrito, Eudosso, Dicearco, Eforo e molti altri e, dopo di loro, Eratostene, Polibio e Posidonio, anch'essi filosofi. La grande ampiezza e varietà di conoscenze, che è l'unica condizione per poter fare fino in fondo un lavoro simile, è propria di chi è portato a considerare insieme le cose divine e umane, la cui conoscenza costituisce per definizione la filosofia. ${ }^{14}$

Dunque, possedere quella vasta varietà d'interessi, interrogativi e conoscenze tipica del filosofo che perlustra l'intero ricostruendo le relazioni tra le sue parti, ciò costituisce la condizione per poter fare il lavoro del geografo. Ben filosofare significa, in definitiva, porsi nelle uniche condizioni del fare geografia stesso. Ma

13 Al riguardo, cfr. Neve 2004, pp. 47-59, in particolare a p. 51: «Il problema dell'uno e dei molti è $[. .$.$] nel cuore della geografia, anzi è il problema con cui la geografia si è$ sempre confrontata».

14 Strabone, Geografia I 1, 1, in Prontera (a cura di) 1983, p. 5. In questa stessa raccolta curata da Prontera si veda almeno l'intervento di Christiaan Van Paassen dedicato prevalentemente a Strabone, ivi, pp. 227-273. 
Strabone prova persino a essere più preciso, perché non s'intenda una condizione in tutto o per eminenza speculativa:

Sia in rapporto alla vita politica e all'attività di governo, sia in rapporto alla conoscenza dei fenomeni celesti e degli esseri viventi in terra e in mare (delle piante, dei frutti e di tutto quanto è peculiare a ciascuna parte della terra), gli interessi della scienza geografica sono vari e molteplici e si addicono ad un uomo che [...] sia attento all'arte della vita e ai mezzi per raggiungere la felicità. ${ }^{15}$

Valutare le più che probabili tracce aristoteliche e stoiche nel pensiero di Strabone esorbita dai limiti di questo intervento. Neppure si può valorizzare la vastissima erudizione messa a frutto nel suo mestiere di storico. Tuttavia, da quanto abbiamo potuto leggere nell'apertura dei suoi Geographika, almeno questo va tenuto fermo: l'uomo che ponga la propria cura nell'arte di vivere, che non si distragga ma perscruti e soppesi gli strumenti utili a conseguire una vita felice ("vita buona, vita felice"), proprio costui potrà occuparsi convenientemente di geografia. E per ciò non soltanto delle regioni della terra e dei viventi che le abitano in ogni genere e specie, o dei fenomeni che appaiono nella volta del cielo che le sovrasta, ma anche di vita politica e attività di governo. Dunque, per Strabone occorre muovere (assiologicamente-aristotelicamente) dall'/all'intero proprio al fine di ben armonizzarne le parti molteplici. Per dare loro sostanza, realtà piena, al modo in cui gli abitanti delle città possono vivere sostanzialmente felici soltanto se gli obiettivi delle loro condotte individuali muovono dal/al bene comune (ogni teleologia compie circoli, mi verrebbe da commentare), cioè soltanto se sono parte attiva di buone arti di governo. Ogni parte (dell'edificio del sapere tanto quanto della vita) presuppone l'intero. Ma con ogni probabilità ci troveremo più vicini a ciò che pensava Strabone se avremo oltrepassato il tenore di una semplice similitudine. Ai suoi occhi la geografia doveva essenzialmente orientarsi alle necessità della vita politica. E non si è lontani dalla verità delle cose quando si ritenga obiettivo di questa sua stessa opera scientifica il risultare utile agli amministratori provinciali della pax romana augustea. Stralciamo di nuovo un passo:

La terra e il mare che abitiamo costituiscono [...] lo spazio delle azioni umane; piccole imprese si compiono in piccoli spazi, grandi imprese in grandi spazi; ma è lo spazio abitato nella sua totalità, ciò che propriamente definiamo ecumene, che costituisce lo spazio delle azioni più grandi. I più grandi generali sono perciò quelli che possono comandare in terra e in mare, unendo popoli e città in un solo impero e sotto una medesima direzione politica. È chiaro dunque che tutta la geografia si rivolge interamente all'esercizio del potere, disponendo i continenti e i mari alcuni all'interno altri all'esterno di tutta l'ecumene $[\ldots]$. È più agevole impadronirsi di un territorio quando se ne conoscano l'estensione, la posizione, le caratteristiche naturali e climatiche. ${ }^{16}$

15 Ibid.

16 Ivi, p. 10. 
Del mondo, quindi, non della terra parlano in verità i geografi. $\mathrm{O}$ meglio, della trasformazione per la quale la terra diventa ecumene, mondo abitato-territorializzato. Tale divenire costituente è "la cosa stessa" del geografo: energia e impresa del popolamento, della dispersione-pluralizzazione linguistica, culturale, sociale, politica, religiosa. Questo è l'intero geofilosofico.

\section{2.}

Trattando di totalità e parti del mondo, Strabone non differenzia corografia e geografia, che nella sua opera sembrano termini utilizzati in modo affatto sinonimico. ${ }^{17}$ Piuttosto, tale distinzione risale a Tolomeo, alla famosa premessa della sua Geōgraphikè yphégēsis (letteralmente, Guida al disegno della terra - con semplificazione moderna noi forse la chiameremmo "Guida geografica" -, ma più nota come Geografia). Ecco:

La geografia è la figurazione mediante disegno di tutta la parte conosciuta della superficie terrestre con gli oggetti collegati alla medesima. Essa differisce dalla corografia, perché questa distinguendo i luoghi particolari li dimostra ciascuno separatamente... È invece compito della geografia trattare della superficie terrestre nella sua interezza. ${ }^{18}$

Nell'eredità disciplinare del sapere geografico è accaduto che sia andata smarrita la sottolineatura tolemaica del disegno, che invece è essenziale. Si è recepita esclusivamente la diversità degli oggetti raffigurati: per la geografia, tutta l'estensione delle terre conosciute; per la corografia, i singoli luoghi che una carta generale dell'ecumene non può delineare in modo particolareggiato. Ma le cose non stanno semplicemente e propriamente così. Leggiamo:

Mentre la corografia ha bisogno della topographia e nessuno potrebbe fare il corografo senza essere capace di dipingere, la geografia non ne ha bisogno affatto, perché con delle semplici linee e dei contrassegni riesce a mostrare le posizioni dei luoghi e i contorni dell'insieme. Per queste ragioni l'una non ha bisogno di alcun procedimento matematico, mentre per l'altra la matematica è di primaria importanza. ${ }^{19}$

Solo che si ascolti, il dettato risuona limpido, inequivocabile. Senza configurazione differenziale di parti, regioni, luoghi, la carta dell'insieme non può essere completata. Le grandi partizioni del mondo conosciuto e abitato, le chorai appunto, sono necessarie al suo disegno: in ciò non può consistere, dunque, il confine epistemologico che separa raffigurazione corografica e geografica. Compito della

17 Cfr. per es. Strabone, Geografia I 1, in Prontera (a cura di) 1983, p. 16.

18 Tolemeo, Geografia I, 1, F. Cordano 1992, p. 188.

19 Ivi I, 5, in Prontera 2006, pp. 75-82, ora anche in Prontera 2011, pp. 95-104, qui pp. 95-96. 
gheographia secondo l'accezione di Tolomeo sarà sempre il procedere dalla delineazione di parti regionali dell'ecumene alla cartografazione del suo insiemetutto, senza mai poter dare a vedere una qualche totalità indifferenziata. Nessunissima geografia senza conoscenza di chorai, che precisamente nel loro particolare differire per posizione ed estensione permettono di configurare l'insieme delle terre note. Il disegno geografico non può essere privo di regioni-partizioni dell'insieme-tutto, così come quello corografico non equivale d'altronde a una cartografia regionale deprivata di sguardo d'insieme-sull'insieme. Infatti, ogni regione stessa, ogni parte è, per sé presa, intera. È un insieme-tutto. I due punti di vista si processano l'uno nell'altro. Soltanto un'interpretazione irriflessa e sbrigativa può ridurre la loro distinzione a una separazione parti/tutto. Sul punto il passo citato di Tolomeo afferma con nettezza ben altro. E cioè: la corografia visibilizza non tanto parti in luogo del tutto bensì - diremmo forse oggi - paesaggi in quanto "luoghi dipinti", "topo-grafie". La geografia cartografa, la corografia no. Il corografo è pittore-topografo di scorci, e non altro; il geografo è cartografo che matematizza qualsiasi grande o piccola totalità, la quale non "fa quadro" a sé senza dover essere ricalcolata nella totalità della superficie terrestre. Alle sue origini, la corografia è per Tolomeo un'operazione ancora mimetica relativa al modo, al come, alla verisimiglianza topografica. La geografia è invece una operazione cartografico-matematica che rende conto schematicamente degli esatti rapporti spaziali secondo cui le partizioni regionali si ritotalizzano-riterritorializzano nell'ecumene.

Se così stanno le cose, v'è allora una costitutiva dimensione estetica che attiene alla raffigurazione corografica, e prevalentemente a essa - se non addirittura soltanto a essa. Dico "prevalentemente" perché mi pare che anche alla gheographia pertenga una determinata mimesis: di forme in calcolati rapporti. Mi verrebbe da dire: la raffigurazione geografica armonizza i differenziali di posizione ed estensione schematizzati-cartografati «con delle semplici linee e con dei contrassegni». Li in-forma presupponendo la totalità di partizioni e connessioni. Pure questa può essere considerata un'operazione mimetica, messa a registro su quantità e formalità. Perché no, in definitiva? Tuttavia la qualità estetica è certamente e pienamente proprietà della raffigurazione corografica, del tutto disinformata di principi matematici e di esatti rapporti spaziali tra parti. Schizzare corografie è una modalità dell'arte della pittura. Non "regionalizza" più di quanto già non faccia il geografo dedito al mapping: però "topografa" paesaggi, mette in scorcio e fa quadro con quel che c'è, che non è tutto il mondo.

\section{3.}

Proprio qui siamo giunti al punto. Anzi, a un punto di collasso della problematica. Tanto nell'enciclopedia del sapere antico, dove si formano le teorie geografiche e le pratiche cartografiche appena accennate, quanto in quella del sapere medie- 
vale, dove fiorisce l'imago mundi esposta da fig. 1 a fig. 3 in uso ancora all'età dei primi grandi viaggi d'esplorazione e di conquista, l'oggetto della rappresentazione è l'ecumene, la porzione di superficie terrestre conosciuta abitata. Quindi, non è il mondo tout court. Non è tutto il mondo. È piuttosto la regione-mondo di cui si sa l'esistenza perché abitata, in quanto ne hanno fatto esperienza i pochi esploratori e viaggiatori, sui racconti dei quali si basano coloro che la pongono in essere con parole e figure. Cioè quelle credibili rappresentazioni che la fanno sussistere quale collegamento di un insieme (pur sempre parziale) di terre emerse note, abitate. "Abitate" qui significa non soltanto, né prevalentemente, "popolate". Piuttosto, significa che gli umani producono (e riproducono per raffigurazione corografico-topografica o geografica) tale "regione-mondo" in quanto complessità di relazioni ricorsive a livello sociale, economico, politico, culturale. Ovvero, la intramano nel suo costituirsi a connessione-complessità di relazioni molteplici. Proprio queste, tuttavia, non possono essere esibite in quanto molteplici se non sempre al modo di una rete, una forma, una regione - di mondo, appunto.

Mondo fatto, istruito, posto in immagine da discorsi e raffigurazioni basati su orientamenti ma soprattutto su confinamenti cognitivo-epistemologici prima ancora che geografici. Su costruzioni identitarie di società e civiltà, su piani valoriali e accumulazioni simboliche. Come mostrato efficacemente proprio da fig. 1 , lungo tutto il Medioevo sino all'alba della modernità ciò resta però vincolato al signum cristiano, alla sacralizzazione dello spazio e della sua rappresentazione. In tal senso l'ecumene medievale raduna certamente una quantità di relazioni eppure queste non fanno Tutto (come abbiamo visto, il Tutto doveva ricomprendere nella sua circonferenza a raggio infinito l'altro Mondo stesso, l'Aldilà dei confini terrestri, siglato come Paradiso). Furono le poste in gioco (sociali, economiche, politiche, culturali) comportate dall'età umanistico-rinascimentale a sortire l'effetto di una diversa esperienza, e dunque pure di un diverso senso (direzione e significato) dell'unità di quell'insieme. A spingere cioè i confini del mondo oltre la loro precedente fissazione. Sfide rilanciate in Europa, nel giro di pochi anni, da una congiuntura epocale: nascita della cartografia moderna, esplosione del colonialismo, prime accumulazioni del capitalismo.

Ora, ho parlato di un punto di collasso intendendo lo squilibrio, sotto la pressione esterna, della capacità di tenuta dell'impianto speculativo in cui si erano strutturati tradizionalmente i rapporti uno/molti, intero/parti. La figura del filosofo-geografo-cartografo che registra l'aprirsi di questa faglia teoretica prende corpo in età moderna nell'algido Kant (meno il filosofo-geografo auspicato da Strabone, che non una specie di sua controfigura "venuta dal freddo"). Kant il quale, non sempre ce lo si ricorda, insegnò ininterrottamente geografia fisica durante i quarant'anni della sua attività didattica, dal semestre estivo del 1756 sino al suo pensionamento nel 1797. Come è stato fatto osservare, «Kant era un geografo prima di essere un filosofo»: nel suo pensiero critico-trascendentale trasmette «la riduzione della conoscenza alla descrizione della rappresentazione 
geografica, della mappa o carta che dir si voglia» ${ }^{20}$. È vero, pare così anche a me. Qui però voglio coglierne il gesto di pensiero nel preciso focus delle antinomie che costruisce con la dialettica trascendentale della sua prima Critica. Intendo laddove è discussa una volta per tutte in sede teoretica l'idea di mondo come tutto. Meglio, del Mondo ovvero Tutto, omnitudo realitatis. Per Kant è impossibile utilizzare sensatamente tale idea. "Sensatamente" in termini kantiani significa: in quanto concetto dell'intelletto, il quale come sappiamo non può uscire dai «confini immutabili» dettatigli dalla sua insularità epistemica. Il Mondo è extraterritoriale. Esorbita la «terra della verità», è puramente inesperibile e inconoscibile purché pensabile. Pertanto resterà sempre altro dall'ecumene, dai molteplici mondi conosciuti, esperienziali e vitali. Parafrasando Nietzsche, il Mondo sta davvero dietro il mondo. Qui, tra i fenomeni - che siano "parti" o "interi" costituiti di parti -, non v'è Tutto. Non è di questa terra, e perciò sfugge anche qualsiasi approccio estetico. Forse lo accarezza un sogno indecifrabile: ma come un'aerea, pura eccedenza noumenica.

\section{4.}

Del Mondo-Tutto non vi sono state soltanto presupposizioni teoretiche e mitologie. Non è apparso esclusivamente in un sogno epistemico. Non l'ha cercato solo l'audace impresa cognitiva. ${ }^{21}$ V'è stata e sempre di nuovo ha preso forma, ben più pericolosa nella sua verità effettuale, l'aspirazione al Mondo-Tutto su di un piano filosofico-politico. Un ossessionante progetto di dominio globale. In termini classici, utilizzando ancora il lessico della filosofia prima aristotelica ma per visualizzare una direzione contraria rispetto a ciò che questa propriamente detta, sarebbe come se si trattasse non più dell'intero che contiene le cose particolari in modo tale che esse siano-predichino uno, bensì come se una sola di queste pretendesse di ergersi essa stessa a intero dopo aver frantumato l'unità di elementi coessenziali della quale partecipa in via costitutiva. Mi domando se in una spinta di questo tipo sia fuori luogo riconoscere l'impulso a una totalizzazione in grado, quando incontrollato, di degenerare effettivamente in concezione totalitaria se non addirittura totalitaristica (com'è accaduto in politica ogni volta che lo stato abbia preteso di investire la totalità della vita dei singoli). È chiaro che si devono dare altre condizioni, ora teoriche ora concrete, perché ciò possa verificarsi. Ma la

20 Farinelli 2003, p. 32. Ma in merito si veda anche il saggio Farinelli 2004 (ristampa anastatica che riproduce in facsimile la traduzione di Augusto Eckerlin per l'edizione stampata dalla tipografia Giovanni Silvestri, Milano 1807-1811, 6 vol., ora in 3), vol. I, pp. I-XXIX.

21 Impresa filosofica che non si conclude certo con le antinomie del disinganno dialettico-trascendentale kantiano: basti pensare a Hegel, al suo rimettere in circolo tramite la dialettica il Tutto come spirito - cioè crescente complessità relazionale. 
realtà della storia continua a esibire prove inconfutabili, e tragiche, di eccessi di tale specie.

\section{1.}

Un disegno analogo sembra attraversare in filigrana la ricezione di Gn 10,1-32, pagina interpretata in senso etnologico-politico e nazionalistico lungo un determinato filone della tradizione storico-religiosa. In alcuni casi influenti dell'esegesi giudaica antica (a. Libro dei Giubilei [II sec. a.C.]; b. Apocrifo della Genesi [I sec. a.C.]; c. Antichità Giudaiche di Flavio Giuseppe [I sec. d.C.]; d. Liber antiquitatum biblicarum [I sec. d.C.]), il testo di Gen 10, che di per sé si limita a far emergere una concezione del mondo ispirata a una «geografia neutral», è infatti diventato strumento per una mappatura ideologica con «conseguenze incalcolabili non solo sul piano delle moderne classificazioni etniche ma anche su quello delle valutazioni antropologiche e teologiche che vi sono collegate, dovute appunto alla nozione di geografia di cui sono una diretta applicazione» 22 .

Non si può non dar corso a un rilievo così importante emergente nelle analisi dei veterotestamentaristi che si sono occupati del quadro storiografico e criticoermeneutico. In generale, si tenga sempre presente che l'Israele di cui parlano le Scritture proviene da una visione elaborata in tempi ben posteriori rispetto alla storia reale di cui si narra e, soprattutto, per finalità di ricostruzione identitaria, di credibilità presso i contemporanei. Finalità emergenti su un piano problematico consegnato alla memoria collettiva e che restano da riconoscere come tali, nella loro ricercata efficacia politica. Ora, in particolare, il caso del commento di Gen 10,1-32 stilato dal Libro dei Giubilei pare molto significativo proprio perché la sua esegesi, appesantita dall'impronta dell'operazione ideologica di totalizzazione-territorializzazione, divenne normativa per le interpretazioni successive sia in alveo giudaico sia in quello cristiano. Naturalmente non è affatto opportuno richiamare per esteso in questa sede l'elaborazione che Giubilei sovrappone al testo masoretico ebraico, tuttavia, anche se molto rapidamente, si può gettare uno sguardo fra i suoi elementi.

La critica, cui dobbiamo rimandare per l'onere della prova, mette in luce alcune precise, influenti linee di tendenza. Mi basta riprenderne tre, diciamo a maglie larghe. Anzitutto l'attitudine a «pensare una geografia reale e dettagliata» e a concretizzarla «etnicamente ed assiologicamente» (invece di quella ancora in larga parte mentale-immaginaria del testo originale); in secondo luogo, la disposizione a combinare palesemente sul piano della registrazione cartografica, ma implicitamente su quello storico-religioso, scienza geografica greca ed esegesi giudaica nell'intento di affermare la centralità di Israele, definito 1'“ombelico della terra", e di situare invece i popoli circostanti «su altri piani (geografici e

22 Prato 1999, p. 90. 
valutativi)», ovvero «in funzione (territoriale e climatica) subordinata»; infine, in virtù proprio di questa esegesi di Gen 10,1-32, l'avvio di

una cosmografia (e anche una corrispondente cartografia) che sarà determinante per gli sviluppi patristici e cristiani e anche per la scienza linguistica ed etnologica successiva e moderna (i popoli e le lingue suddivisi in semiti, camiti e "iafetiti" [ottenuti per esclusione!] la quale quindi non è direttamente "biblica", ma è mediata essenzialmente dal giudaismo. ${ }^{23}$

Dunque, il rimaneggiamento esegetico-ermeneutico del testo masoretico ha sfruttato i dati geografici via via resisi disponibili con l'obiettivo di avvalorare e legittimare le territorializzazioni dell'ecumene. La sua proiezione-realizzazione cartografica, di fatto incontestabilmente performativa, è stata strumento per concludere a pesanti giudizi sulle popolazioni. La mappa neutrale-mentale implicita in Gen 10 ha assunto gli effettivi tratti di una geografia reale disegnata attorno a un centro (Gerusalemme) eletto espressamente come tale, non più sottinteso per sola necessità di computo geometrico. La "tavola delle nazioni” è potuta servire così a strategie di totalizzazione tramite le quali il gruppo discendente da Sem ha voluto imporre l'intangibile sacralità di Gerusalemme e l'universalizzazione del suo spazio sfruttando una proiezione gerarchica in verticale.

Secondo la mappatura che il Libro dei Giubilei fornisce combinando geografia greca ed esegesi giudaica, le tre parti della terra assegnate alla successione dei figli di Noè possiedono qualità climatiche diverse distribuite lungo l'asse verticale che attraversa una zona mediana temperata. Non a caso l'area toccata a Sem, cioè quella interna e più mite caratterizzata da una migliore abitabilità, si trova al centro di tale zona temperata - mentre a Iafet spettò l'area settentrionale e a Cam quella meridionale-occidentale. Di qui proviene la spiegazione stessa che ha fatto risalire l'assetto geopolitico dell'Europa moderna alle migrazioni sviluppatesi dall'età antica lungo l'intero Medioevo, avendo voluto riconoscere genealogicamente $\mathrm{i}$ discendenti di Iafet proprio in quelle popolazioni in movimento. La questione migratoria si troverebbe pertanto allacciata in modo essenziale, mitologicamente o storicamente poco importa, alla tripartizione della superficie terrestre e alla conseguente invenzione dell'idea di nazione (in un'accezione necessariamente ancora generica, come ho già detto quasi soltanto linguistico-culturale e riferibile di per sé a qualsiasi forma di raggruppamento o comunità, dunque ancora distante dall'indicare il fondamento del potere politico poi convergente nell'unità statale). 


\section{2.}

Non v'è invenzione d'idea che non comporti quasi simultaneamente la ricostruzione della sua stessa storia, per quanto discontinua e multiplanare questa debba pur essere schizzata. Materialmente, la storia dell'idea di nazione si scontorna anche grazie alla designazione degli antenati. Ogni nazione riconosce sempre i propri padri fondatori e proietta le esistenze individuali nella trama del racconto collettivo iniziato dai padri. Venera coloro che hanno impresso un ethos come un passato immemorabile ma vincolante. Cioè che hanno radicato qualità e condotte morali, forgiato un linguaggio e una cultura, iniziato la loro tradizione e determinato un modo di abitare il mondo, di farne esperienza. È così che prende impulso l'individuazione genetica di un'identità precisa e riconoscibile, la coscienza di un patrimonio identitario di valori spirituali-culturali tipici ed esclusivi di un soggetto collettivo.

In tal senso mi pare che si possa leggere anche l'operazione immanente di Gen 10,1-32. Tuttavia, la storia politico-religiosa della sua esegesi e del suo tramandamento, con la cartografia del popolo eletto delineatasi già a partire dal II secolo a.C., mostra come quell'antica formulazione dell'idea di nazione, per dir così ante litteram, sia servita a giustificare ciò che dall'età moderna abbiamo imparato a chiamare "nazionalismo" - solitamente si dice: a partire da Rousseau e con gli ideali esportati dalle guerre della Francia rivoluzionaria e napoleonica. Un nazionalismo cioè quale ideologia per l'assoggettamento di altri popoli, per una politica di potenza e l'imposizione di una supremazia. Certamente non in quanto, al contrario, grido di liberazione di un popolo assoggettato. Ora, se così stanno le cose, viene da chiedersi quanto sia corretto continuare a parlare di "tavola delle nazioni". Non è prevalsa forse una sorta di "tavola dei nazionalismi etnopolitici”, filiazioni molteplici giustificate da un'unità originaria? Qualsiasi risposta s'intenda far seguire a tale interrogativo, mi preme ribadire che la nascosta potenza della mappa implicita nel testo masoretico consiste nel valere piuttosto da matrice di dispersioni molteplici, di pluralità persistenti nei loro movimenti anomali, di divenire moltitudinari, di popolamenti deterritorializzanti.

Questione di rilievo certamente non trascurabile, anzi, d'importanza cruciale ancora ai nostri occhi. Nella luce di quei giudizi ispiratori di valutazioni ideologiche circa l'elezione dei popoli si può vedere rifratta l'acuta miopia attuale delle politiche migratorie europee, le chiusure nazionalistiche, il selvaggio riprodursi di scatti xenofobi, la moltiplicazione di normative volte a bloccare e respingere $\mathrm{i}$ movimenti di donne, uomini, bambini in fuga da contesti di guerra e sofferenza. A tale proposito dovrei condurre verso nuovi piani di sviluppo problematico quanto ho già iniziato a trattare altrove $:^{24}$ non è questo tuttavia il mio attuale obiettivo. Nei paragrafi che mi restano prima di concludere intendo invece focalizzare

24 Cfr. Boffi 2014a. 
l'attenzione su alcune immagini tratte, prima, dall'arte figurativa e, poi, da un lavoro filosofico-letterario.

\section{3.}

Guardo a un'opera prodotta da "Société Réaliste", collettivo artistico nato nel 2004 per iniziativa di Ferenc Gróf et Jean-Baptiste Naudy. Un sodalizio che ha realizzato vari progetti declinando un paradigma geoestetico in diverse pratiche dell'arte. Le sue ricerche si sono indirizzate sulle relazioni del singolo e delle istituzioni con il territorio, sull'interpretazione e l'uso dei modelli di rappresentazione del mondo e di parti di esso, sui processi di trasformazione architettonica delle realtà urbane e ambientali, sulle politiche europee degli spazi e dell'immigrazione, sui legami tra marketing ed economia dell'arte. Ma ad attirare l'attenzione in particolare è un'opera che si trova in un legame intrinseco con tutto il discorso condotto sin qui. Si tratta di un'ulteriore rielaborazione, questa volta in senso critico-ironico, del modello T-O già abbondantemente illustrato.

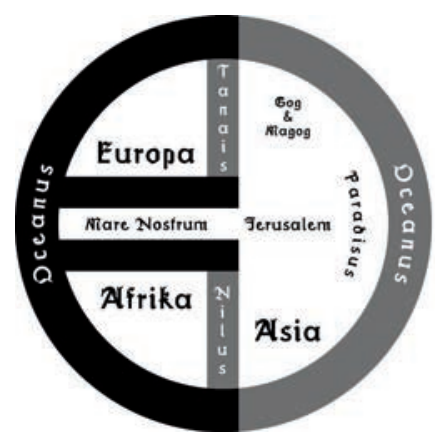

Fig. 4.

EurO\&T (2011): una placca metallica smaltata $(60 \times 60 \mathrm{~cm}$.) in cui l'immagine di fig. 1 appare ruotata di $90^{\circ}$ in senso orario, di modo che il quadrante dell'Europa si ritrova nella sommità di sinistra. Precisamente la metà sinistra della circonferenza è colorata e con essa anche due segmenti, rispettivamente superiore e inferiore al raggio del mare magnum (l'asta della precedente $\mathrm{T}$ ), tanto da farne risultare il simbolo grafico dell'euro. Ecco il punto ironico-critico: l'imago mundi medievale viene così "trasvalutata" in imago Europae e subito "svalutata". Il modello T-O citato dal passato offre un patrimonio identitario nella caricatura "monetarizzata" dell'Unione europea creata dagli Istituti centrali di credito. Questa è la nuova geografia, che sovrappone il conio del mercato unico all'intero dell'ecumene e lo sostituisce così all'ispirazione religiosa dell'antico diagramma. $\mathrm{O}$ forse sarebbe più opportuno commentare: che riconosce, anzi fa ecumene nell'e- 
spansione del mercato capitalistico. Non i grandi valori della tradizione giudaicocristiana, seppure spesso sbandierati quali inalienabili radici: ma la moneta unica.

$\mathrm{Al}$ clamoroso panico per la perdita dell'identità l'opera risponde con un'operazione artistica che probabilmente avrebbe divertito molto anche il buon vecchio Aby Warburg. L'avrebbe messo alle prese con una sopravvivenza del passato capace di reclamare la propria presenza nella formidabile sinossi per immagini della memoria culturale dell'Occidente quale fu il suo incompiuto atlante Mnemosyne. Avrebbe potuto essere collocata, ironicamente - cioè anche quale forza d'interrogazione -, tra le prime tavole del suo Bilderatlas, forse persino in un controcanto alla tavola (A), dedicata ai diversi sistemi di relazione in cui l'uomo si trova inserito. ${ }^{25}$ Quale più efficace sistema attuale di relazioni, fuori da quello di occupazione della terra da parte del mercato capitalistico e della ricchezza finanziaria digitalizzata? Quale altra potente forma di organizzazione-unificazione di movimenti e molteplicità? Questa è, di fatto, l'ecumene che sfortunatamente, barbaramente abitiamo - per molti senza mai poterla sentire propria, eccetto quale forma d'immiserimento e sofferenza.

Risibile e feroce Europa delle banche, sempre meno unione di parti coessenziali al tutto. Anzi, Unione sempre più disunita, disgregata, sull'orlo del collasso. È divenuta simbolo della Fortezza eretta a svantaggio di poveri, stranieri e migranti, di quei "frammenti in movimento" i quali continuano a costituire null'altro che singolarità qualsiasi ogni volta scartate, gettate via proprio perché in movimento - mai partecipi dell'abbraccio di un intero, né identificabili in una delle sue parti. Nessuna voce di Europa unita è possibile sentire a loro sostegno, al loro fianco: ma soltanto qualche isolato, indignato richiamo mediatico nel putiferio assordante di strilla che più parti alzano. L'Unione è stata pensata forse sin troppo sul modello del precedente Stato-nazione, soltanto esteso su scala continentale. Nei termini illustrati prima, forse potremmo trovare una glossa a tale fallimento di qualsiasi disegno strategico unitario di una territorialità aperta, transnazionale e transfrontaliera, scrivendo di una geografia di poteri che cancella topografie-corografie di spostamenti, erranze, nomadismi, apolidie, migrazioni.

In una totale competizione reciproca fra i Paesi membri dell'Unione europea, ogni minima parte pretende di legiferare e comandare valendo di per sé, agitando brutalmente la questione delle autonomie o delle indipendenze, insomma - diremmo - della propria particolarità pretesa a valore assoluto. Ognuna si chiude nella propria narrazione identitaria-nazionalistica, quasi sussistesse da sola al mondo. Anzi, essa $f a$ il proprio mondo nel chiuso della sua cerchia di violenta autoreferenzialità ma prontissima a imporlo esternamente a cerchie sempre più estese, in una pretesa significazione e validità universali. Si tratta di politiche di supremazia del proprio particolarissimo mondo esercitate da parti e controparti

25 Mi sono occupato di questa tavola in Boffi 2014b, pp. 200-201; più complessivamente sull'Atlante warburghiano, ivi, pp. 41-60: ma Warburg costituisce un punto focale di tutto il volume. 
incessantemente nell'esigenza di valere come fosse in gioco non la propria parzialità bensì l'intera globalità del Mondo-Tutto. $\mathrm{O}$ meglio, si tratta dell'esplicita, programmatica rivendicazione di condurre totalmente le politiche di parte in quanto compiute in se stesse. In tal senso potremmo commentare: con l'intera pretesa validità su tutto, e in ogni caso prevalendo su altri. Politiche in quanto universali, insomma - e non invece per quel che veramente sono, sempre soltanto misure "particolari", "locali", "regionali".

Oggi l'unico universalismo sussistente a tutti gli effetti è l'imperativo del mercato economico-finanziario globalizzato, adattato alla sfericità telematica del mondo. Esercita ovunque una potenza totalizzante e riterritorializzante, travolge tutto da parte a parte, su una piazza planetaria dove s'interagisce materialmente e immaterialmente, si acquistano e si vendono flussi di dati, di beni, di informazioni. Possiede - nei termini del testo ebraico che racconta la "torre di Babele" - «un solo labbro» e «parole uniche», cioè unità omogenea di linguaggio. Unità di impero su tutto il mondo. Ma è poi davvero soltanto questo il mondo, quello che le parole d'ordine della crisi stanno facendo funzionare mettendo in stato di debito intere popolazioni dall'inizio degli anni 2000? Fuori da questa implacabile forma di governamentalità, non c'è altro mondo, possibile o reale che sia?

\section{5.}

Chiamo mondo-tutto il nostro universo per come cambia e perdura cambiando e, al contempo, la "visione" che ne abbiamo. La totalità-mondo nella sua diversità fisica e nelle rappresentazioni che essa ci ispira: che non sapremmo più cantare, dire né lavorare con sofferenza a partire dal nostro solo luogo, senza precipitare nell'immaginario di questa totalità. I poeti l'hanno presentito in ogni tempo. ${ }^{26}$

L'immagine di mondo-tutto al centro della nostra questione ritrova un'eco potentissima nella poetica e nella riflessione di Édouard Glissant. ${ }^{27}$ Vi risuona per immagini e per concetti, nella caratteristica indisgiungibilità di teoria, immaginazione, scrittura. Durante un'intervista rilasciata a Lise Gauvin il 17 ottobre 2006, subito dopo la pubblicazione di Una nuova regione del mondo, primo e unico

26 Cfr. Glissant 1997, p. 176. Opportunamente le versioni italiane da Glissant mantengono le iniziali maiuscole presenti nell'originale francese Tout-Monde, con le quali l'autore intende sottolineare il funzionamento categoriale del concetto entro il suo discorso. Tuttavia, per distinguere tale idea di Glissant da quella ontologico-metafisica della tradizione filosofica continentale, che le è alternativa e che ho richiamato contrassegnandola a mia volta con le maiuscole di "Tutto", "Mondo", "Mondo-Tutto", ho preferito ritrascrivere il termine di Glissant con le minuscole. Dunque, da qui in poi sarà: "mondo-tutto".

27 Mi è già capitato di soffermarmi sul pensiero di Glissant in Boffi 2014b, in particolare al Capitolo secondo della seconda Parte, pp. 148-166. 
volume della sua Estetica, Glissant ha avuto modo di illustrare di nuovo il concetto: «è una visione del mondo, ciò che chiamo il mondo-tutto, che è non una parte del mondo ma una regione del mondo nella quale entriamo tutti allo stesso tempo. È ciò che caratterizza la sua novità» ${ }^{28}$. E guardando a Deleuze, autore molto caro e fonte di non pochi stimoli:

Che cos'è la totalità-mondo, che io chiamo anche mondo-tutto? Mi sembra che una delle possibili risposte in Deleuze sia questa: è un mondo in cui «si entra in zone di vicinanza, piuttosto che acquisire dei caratteri formali». Semplicissima e profonda intuizione di ciò che per noi oggi è cambiato. ${ }^{29}$

Tout-monde, mondo-tutto è uno dei cardini, delle idee-chiave del suo pensiero, cui Glissant dedica sia un romanzo sia un saggio. ${ }^{30}$ Per suo tramite pensa lo stato attuale della mondialità (di contro e in lotta con il suo «disastroso rovescio», ciò che lui chiama «mondializzazione» e noi siamo soliti dire globalizzazione). Mondo-tutto è la condizione del mondo come s'è realizzata nella nostra epoca: «un mondo che per la prima volta e così realmente e in modo tanto immediato, folgorante, si concepisce contemporaneamente molteplice e uno, e inestricabile» ${ }^{31}$. Inestricabile è la prossimità che si genera nello spazio (tra gli spazi) per il moto di singoli e gruppi che, al pari di cariche elettriche variabili ma sempre presenti nel tempo, determinano zone di contatto, veri e proprio campi magnetici. Tuttavia non soltanto nello spazio e tra gli spazi. La condizione di mondo-tutto "fa entrare in condizioni di vicinanza" generandole anche nel tempo e tra i tempi. Mentre sino a qualche secolo fa gli archi temporali che distanziavano le culture erano immensi, e le influenze tra l'una e l'altra cultura, le trasformazioni dall'una all'altra anche sullo stesso suolo geografico richiedevano tempi lunghissimi, oggi avvengono invece in una presente molteplicità fatta di intrecci temporali diversi, di contemporaneità improvvise, così da risultare immediatamente percepibili per quello che sono e che comportano.

Mondo-tutto: tale neologismo glissantiano ritrae il volto di ciò che prima ho detto complessità di relazioni ricorsive a vari livelli (sociale, politico, economico, culturale, spaziale, temporale). È il mondo nelle sue diverse località e qualità materiali. Il nostro unico mondo dell'esperienza, delle forme di vita e dell'essere comune, necessariamente sempre ad un tempo uno e molteplice. "Ad un tempo" significa: nello scambio fulmineo tra unicità e molteplicità. Mondo che si concretizza quindi non in un costrutto statico, bensì in un tourbillon caotico di velocità e di ritmi, di cadenze, gesti, soprassalti, voci, erranze, migrazioni, popolamenti, esplosioni. Non riduttivamente uno, non disgregatamente molteplice; non

28 Glissant 2010, pp. 88-89 (corsivo mio).

29 Glissant 2008, p. 128.

30 Rispettivamente Glissant 1993, Glissant 1997.

31 Glissant 2008, p. 15. 
unitariamente tutto, non moltiplecemente caos: ma e l'uno e l'altro - e insieme né l'uno né l'altro.

La condizione di mondo-tutto appartiene a ogni essere che vive. Preso in una trama relazionale mobilissima di scambi e avvicendamenti per il solo fatto di esistere, di essere venuto al mondo, ogni singolo vivente (uomo, animale e pianta) si trova nell'inestricabile "unimolteplicità" - immanenza. Non può non esserne compenetrata in modo costitutivo e permanente la stoffa del suo essere. Ne è pervasa ogni sua fibra. Il mondo è dei viventi, ma ogni vivente è mondo per come il mondo diviene lì dove questo stesso vivente è venuto al mondo. Essere lì, nella località e però in connessione a tutti gli orizzonti - di nuovo: immanenza. Presente in tutti i sensi secondo un'infinita variazione di differenze: localmente uno e molteplice, inestricabilmente messo in gioco dal congiungersi-disgiungersi, anche violento, degli uni e degli altri.

Il pensiero che penetra tale condizione di mondo-tutto è «arcipelagico»-e forse non può non esserlo per Glissant, nativo della Martinica. Arcipelago è l'orizzonte nativo, vitale, poetico, filosofico. Questo orizzonte non allinea, è brulicante. Vi lussureggiano centri. Extraterritoriale rispetto al pensiero territoriale europeo, è costitutivamente aperto, policentrico, multiplanare, ancora una volta in un senso molto vicino ai millepiani di Deleuze e Guattari.

Quest'apertura, da luogo a luogo, tutti ugualmente legittimati, e ognuno di loro in vita e in connessione con tutti gli altri, e ognuno di loro riducibile a qualsiasi cosa, è ciò che informa il mondo-tutto. Noi interroghiamo questa nozione di luogo (i Mille Plateaux), i suoi limiti e le sue aperture, come fosse lo strumento più sicuro, precisamente o molto oscuramente, per nominare ognuno dei nostri luoghi e per collegare tutti i luoghi tra loro. $[\ldots]$

È una scelta poetica di Deleuze e Guattari, quanto a me, mi soffermerei forse a pensare Mille Jungles o Mille Cyclones, ma il fondamento è lo stesso: laddove le geografie delle idee, dei desideri, della creatività, sfuggono al Territorio, ai sistemi continentali ed entrano negli Arcipelaghi. Le imbarcazioni nude navigano sulle savane, la canna da zucchero cresce in mezzo alle onde del mare. ${ }^{32}$

Mi sembra molto chiaro cosa intenda Glissant, il quale torna sul punto della distanza dal pensiero continentale europeo quasi in un aforisma: «Pensiero continentale, che svela in diaspore gli splendori assoluti dell'Uno. Pensiero arcipelagico, in cui si concentra l'infinita variazione della Diversità. Ma la loro alleanza è ancora a venire» ${ }^{33}$. Il pensiero arcipelagico vibra come «pensiero del tremore», «distrae e spinge alla deriva le imposizioni dei pensieri di sistema» ${ }^{34}$. Trema nei tremori dei viventi presi nei giochi di inclusioni ed esclusioni sociali. «Si oppone al

$\begin{array}{ll}32 & \text { Ivi, p. } 129 . \\ 33 & \text { Ivi, p. } 217 . \\ 34 & \text { Ivi, p. 73. }\end{array}$


pensiero brutale, duramente univoco, dell'io a esclusione dell'altro» ${ }^{35}$. Pensiero che diffrange nei sapori inattesi della complessità, inarrestabile e irriducibile. Diffrange il mondo-tutto, «che è totalizzante senza essere (per noi) totale» ${ }^{36}$. Totalizzante nel senso che continua a produrre interi erratici e reticolari, a mescolare e diversificare totalità frammentarie, senza compiersi mai per noi. Mai tutto compiuto, rappresentato, cartografato, misurato, posseduto, venduto l'assetto definitivo del mondo non sussiste. Il mondo-tutto è dismisura: without mapping.

\section{1.}

Il mondo trema, si creolizza, cioè si moltiplica, mescolando le sue foreste e i suoi mari, i suoi deserti e le sue banchise, tutti minacciati, cambiando e scambiando i suoi costumi e le sue culture e quelle che ancora ieri si chiamavano le sue identità, in gran parte massacrate. Il pensiero arcipelagico trema di questo terremoto, sconvolto da queste crisi geologiche, attraversato da questi sismi umani, tuttavia riposa presso fiumi che finalmente si acquietano e lune che si attardano languide. Ma questo pensiero non è fatto di un unico impeto indistinto né di un tuffo sordo nel profondo, esso progredisce in reti che si attraggono e che non abbandonano alcun dato del mondo lontano. Esso introduce a quella che Montaigne chiamava "la forma intera dell'umana condizione", la forma, non l'Uno, né un'essenza, ma una Relazione in una Totalità. ${ }^{37}$

Il pensiero del mondo-tutto è pensiero delle reti-relazioni. La caotica, tremante totalità-mondo si riflette nella forma-relazione, che le è propria - è $i l$ suo proprium. L'intero è relazionale, in altri termini. È così perché $\grave{e}-$ che fallisca, che ceda, che esulti, che trionfi, che cresca, imploda o s'espanda. Il pensiero vi si muove per tracciati anomali e frattali di sistemi erratici, per faglie, precipitazioni temporali e vortici improvvisi di popolazioni. Lo insegue, lo pensa necessariamente dall'interno: "disperdendovisi", diffrangendosi il più possibile per tutte le sue trame in divenire, lungo la superficie cangiante dove s'incidono i suoi movimenti, negli spazi-tempo delle culture che lo hanno abitato e lo abitano, che vanno preservate nelle loro peculiari diversità. Non può prendere forma se non come pensiero reticolare-relazionale. Pensa la dispersione mondiale che affligge l'umanità dai tempi dei figli di Noè. Si moltiplica in pensieri del popolamento, delle moltitudini, del diverso, della processualità, del policentrismo, del tremore e della dismisura. Scaturisce dall'interazione fra concetti che esso stesso inventa e a sua volta fa scaturire in processi perpetui di apertura, d'ingresso reale a ciò che Glissant - si badi da un punto di vista antropologico e nient'affatto linguistico chiama "creolizzazione". «Il mondo-tutto è quindi il luogo di una realtà pro-

35 Ibid.

36 Glissant 1997, p. 22.

37 Glissant 2008, p. 73. 
cessuale, che io chiamo creolizzazione. Oggi, questo processo mescola le contrazioni di spazi e le precipitazioni di tempo, e le sue risultanti sono inattese.» ${ }^{38}$

Fra tutti i concetti forgiati da Glissant, cui l'inventiva teorica e linguistica certo non ha fatto difetto, risulta decisivo quello di "Relazione" (nelle prime opere scritto con l'iniziale minuscola e con la maiuscola in quelle successive, una volta guadagnata maggior consapevolezza categoriale). Decisivo in quanto vi muove e ne dipende il pensiero del mondo-tutto. Vi muovono l'intero procedere poeticometapoetico e la potente critica che Glissant porta alle filosofie dell'Uno, dell'Identità, dello Stato-nazione continentali, nonché al loro referente geograficopolitico: l'Occidente della Conquista. Tramite l'operatività concettuale della elazione, la totalità mondo è sempre rilanciata nel divenire turbinoso delle sue molteplici singolarità e diversità. Tutto è "relativo" nel senso di "relato-a", correlato. La Relazione ha bisogno delle differenze ed effettua il diverso. Non verticalizza all'Uno bensì depista mentre appaia; pone di traverso e in orizzontale; accomuna mentre disorienta nella moltiplicazione dei centri: al modo in cui accade l'esperienza spaziale delle differenze specifiche che fanno "arcipelago", le quali spingono l'una all'altra, isola dopo isola ma anche isola con isola. Senza radice unica, senza origine prima, senza identità esclusiva. «Quando consideriamo le nostre differenze tra noi e il mondo, diventiamo intolleranti, integralisti in rapporto a noi stessi e alle nostre credenze. [...] La differenza non è ciò che ci separa. È la particella elementare di ogni relazione. È tramite la differenza che funziona ciò che chiamo la Relazione con una $\mathrm{R}$ grande.» ${ }^{39}$

Il tenore etico-politico di quanto abbiamo appena letto è inequivocabile. La Relazione non si esaurisce in una categoria poetico-filosofica o in una riflessione metapoetica. La Relazione è modo d'essere al mondo. Modo d'essere di quel mondo-tutto che è simultaneamente caos-mondo. Modo di essere-comune sorgivamente aperto e incompletabile, intrecciato, multiplo, fatto da differenze ognuna delle quali possiede variabili infinite. La nuova regione del mondo è l'immanente raccordarsi di un insopprimibile multiregionalismo. Si apre ai viventi come relazione di differenze-singolarità che rimangono nella loro pluralità immanente e nell'immanenza di un identico che non le omogeneizza né le neutralizza nella medesimezza dello "stesso". Nella "Nota all'edizione italiana" (2007) della Poetica della Relazione ${ }^{40}$ Glissant torna a ribadire il punto di vista esposto poco prima nell' Estetica (2006): «La particella elementare dell'identità non la concepiamo più entro lo stesso, ma nel gioco delle differenze, scoprendo con stupore che le nostre identità giocano il gioco delle differenze, tanto almeno

\footnotetext{
38 Ivi, p. 129.

39 Glissant 2010, p. 91.
}

40 Giusto per capire come si raccordino le date: il testo originale della Poetique de la Relation è stato pubblicato in Francia già nel 1990, dunque molto prima dell'edizione italiana. 
quanto riposano sull'immanenza dell'identico. In questa nuova regione del mondo le differenze non contrappongono, raccordano» ${ }^{41}$.

\section{2.}

Queste osservazioni di Glissant si mettono nella prospettiva di «un'Estetica del mondo, $[. .$.$] un sentimento della nostra relazione con la bellezza, concepita come$ forza di rinnovamento della nostra presenza nel mondo ${ }^{42}$. Un'estetica politica. Politica precisamente nell'esigenza di salvare le bellezze del mondo che, nei diversi beni comuni materiali e immateriali, costituiscono il bene comune da condividere e trasmettere alle generazioni venture. Lo è interamente per noi, politica. Non può in alcun modo attenere alle organizzazioni socio-economiche delle nostre esistenze, alle forme strutturate di comando e gestione delle nostre soggettività in vista di determinati obiettivi. Lo è entro un contesto il quale, nella messa in gioco delle forme culturali e ideologiche che ne hanno presieduto la costruzione postmoderna, ha dissolto programmaticamente la qualità ontologicopolitica del mondo.

Ciò che qui Glissant chiama "estetica del mondo" s'iscrive sugli orizzonti del campo geoestetico. La geoestetica è potenza deterritorializzante e diasporica rispetto all'ecumene capitalistica. Forse non riesce a impedire che la terra continui a trasformarsi in territori di mercato, in reti digitali della finanza, ma può sottrarle regioni e memorie ancora da farsi. Da difendersi in comune. È brulicante e cresce con un comune gesto resistenziale che s'intrude, rompe, raccorda in connessione a tutti gli orizzonti, gli immaginari, nel vortice di tutti i tempi. Nell'edizione originale della Poetica della Relazione Glissant aveva definito "estetica della terra" ciò che nella posteriore Nota alla versione italiana dello stesso testo rinomina non a caso "estetica del mondo":

Estetica della terra? [...] Sì. Ma estetica del rovesciamento e dell'intrusione. Trovare dei febbrili equivalenti per l'idea "ambiente" (che io preferisco chiamare "l'intorno") e per l'idea "ecologia", che sembrano così oziose in questi paesaggi della desolazione. Immaginare forze che sanno di brace e di sciroppo dolce, per l'idea di amore della terra, che è così ridicola, o che spesso fonda intolleranze settarie. Estetiche della rottura e del raccordo. $^{43}$

Sostenere che il pensiero geoestetico del tremore si rintracci a partire dai giorni della dispersione, dopo il diluvio, dopo Babele, in prospettiva geoestetica significa lasciare venire un'apertura infondabile (un senza fondo) di vita plurale che eccede

41 Glissant 2007, p. 7.

42 Ibid.

43 Ivi, pp. 141-142. 
i contorni di qualsiasi mappa, una potenza di popolamento, un'interminata narrazione corale di differenze. Significa che il pensiero di Totalità-Tutto è ancora (kantianamente) inarrivabile. Che questo mondo - con il carico penoso dei suoi "terzo", "quarto" mondo ecc. - non è Tutto. Non lo è non soltanto dal punto di vista epistemico ma anche nella sostanza materiale, nella contingenza delle cose, nelle congiunture locali di ciò che facciamo e che capita, nell'intreccio di spazi e durate reali.

Ciò implica che anche il "solo labbro" e le "parole uniche" del mercato non dovrebbero totalizzare senza resto né rimedio l'essere comune. L'ecumene oggi è espansione del mercato universale, senza altro discorso e disegno entro l'impero della sua globalità. Hanno forse ragion d'essere univoche, però, la sua geografia, la sussunzione reale di ogni luogo e società sotto il capitale finanziario, la neutralizzazione capitalistica di possibilità alternative di relazione? Non hanno ragion d'essere univoche, si deve rispondere, per quanto costituiscano la ratio effettiva, l'involucro e l'impianto della formazione economico-sociale dominante, dell'impoverimento di popolazioni, culture, civiltà, linguaggi, filosofie, arti, forme di vita. Nuovi popolamenti materiali e mentali possono affacciarsi. Perché ciò accada, occorre però che si mantengano distanza e apertura. Occorre che le geografie esistenziali si fecondino con le geografie immaginarie in forme nascenti di libertà, ovvero quale potenza e costituzione di nuovo essere, per ogni luogo possibile di cooperazione e d'intreccio. Occorre "lavorarvi" da un luogo all'altro, in una paziente ma resistente costruzione politica (e perciò geografica) degli spazi e dei tempi relazionali dell'esistenza.

\section{3.}

Un pensiero geoestetico avverte sensibilmente che ci si debba astenere dall'inseguire i disegni culturali, sociali, economico-politici non soltanto del tuttomercato ma altrettanto delle particolarità che, ergendosi contro una tale totalizzazione, proprio per separarsi da essa, si pretendano a loro volta Tutto. Nessun radicamento etnico, culturale, politico in Mondo a parte. Ogni mondo a parte, cui rischiano di culminare anche i più intransigenti slanci radicali d'indipendenza e autonomia dall'insieme, produce soltanto guerra civile, povertà, divisione, separatezza, apartheid. Ogni radicamento identitario, culturale, religioso, etnico, economico, nazionalistico nella propria territorialità culmina in bellicosa segregazione. Comporta povertà e violenza, soprattutto a chi non può difendersi. Ora, nel mondo sempre più globalizzato, quale guerra esportata o importata (appunto: culturale, religiosa, etnica, economica, nazionalistica) non è già "guerra civile", come iniziò a esserlo sotterraneamente già la lunga guerra fredda del Novecento? A quale conflitto internazionale o nazionale ci si può sentire estranei? Una neutralità è lecita e giustificabile più di un intervento? 
Semmai vi sia guerra da scatenare, è piuttosto una lotta gioiosa, pacifica ma incessante in ogni regione del mondo, senza parti escluse, ogni giorno, contro la sussunzione della totalità della vita e delle sue forme alle reti materiali-immateriali dei poteri. Fare guerra totalmente al feroce saccheggio della terra. All'immiserimento culturale ed economico; alle facili idiozie sul cosiddetto "scontro di civiltà"; alla messa in stato di debito della vita materiale di quasi ogni lavoratore; agli interessi dei pochissimi che giocano a scacchi con i destini, le fortune, le sostanze di moltitudini planetarie. Pensare presentemente il mondo non è possibile senza lottare per tutelare i diritti delle generazioni venture, di tutta la comunità di vita che comprende ogni vivente sulla terra sino alla biosfera stessa; lottare per tutelare la salute senza discriminazioni oltre il limitato, particolare benessere individuale; lottare contro l'intollerabile schiacciamento di ogni orizzonte di attesa sull'immediato e il precario, contro il progetto di desertificazione degli immaginari, di persistente neocolonizzazione delle esistenze, di agglutinamento delle singolarità e annientamento delle molteplicità.

Geografia o corografia, in quale disegno, in quale scrittura ricade questa prospettiva geoestetica? Anzi, forse è ancor meglio chiedersi: da dove muove? Non dall'Uno né dai molti in quanto molti. Non dal Tutto né dalla parte. Non da esclusivi giochi di totalizzazione-territorializzazione, dovunque provengano: ma dal frammento in movimento che, espulso dal Tutto e rifiutato dalla parte, si sposta, e dall'estremo orlo della nuova regione del mondo, immanente alla terra per il suo incessante popolamento, reclama un luogo per poter abitare, parlare, lavorare, amare in comune. Frammento che migra. Che, sospeso nel movimento migrante, traccia linee di fuga da ogni territorializzazione. Fuori dai contorni fissi di ogni carta - without mapping.

È vero, infine, che in questo mercato aperto, in questo "mondo-mercato", in questo "mercato-mondo", le differenze tra penuria e abbondanza suscitano onde migratorie intense, simili a cicloni che nessuna frontiera riuscirebbe ad arginare. Sapiens è per definizione un migrante, emigrante, immigrante. Per questo si è disseminato, ha percorso il mondo e ha attraversato le sabbie e le nevi, le montagne e gli abissi, ha disertato le carestie per inseguire la possibilità di bere e mangiare. "Non esistono frontiere che non vengano oltrepassate". Questo si verifica da milioni di anni. Sarà così per sempre (ancora di più a causa degli sconvolgimenti climatici che si annunciano) e nessuno dei muri che, con pretesti diversi, si alzano dappertutto, ieri a Berlino e oggi in Palestina, nel sud degli Stati Uniti o nelle legislazioni dei paesi ricchi, saprà fermare questa semplice verità: che il mondo-tutto diventa sempre più la casa di tutti - in creolo Kay tout moun -, che esso appartiene a tutti e che il suo equilibrio passa attraverso l'equilibrio di tutti. ${ }^{44}$

44 Chamoiseau / Glissant 2008, pp. 12-13. 


\section{Bibliografia}

Boffi, Guido: Migrazioni. Note di geoestetica I. Napoli / Salerno 2014a.

Boffi, Guido: Per altri atlanti. Note di geoestetica II. Napoli / Salerno 2014b.

Chamoiseau, Patrick / Glissant, Ėduard: Quand les murs tombent. L'identité nationale hors-la-loi? Paris 2007; tr. it.: Quando cadono i muri. L’identità nazionale fuori legge? Roma 2008.

Cordano, Federica: La geografia degli antichi. Roma / Bari 1992.

Crampton, Jeremy W.: Mapping: A Critical Introduction to Cartography and GIS. Malden / Oxford / Chichester 2010.

Farinelli, Franco: La crisi della ragione cartografica. Torino 2009.

Farinelli, Franco: "Experimentun mundi", prefazione a: Kant, Immanuel: Geografia fisica. vol. I. Bergamo 2004, pp. I-XXIX.

Farinelli, Franco: Geografia. Un'introduzione ai modelli del mondo. Torino 2003.

Glissant, Èdouard: L'imaginaire des langues. Entretiens avec Lise Gauvin (1991-2009). Paris 2010.

Glissant, Èdouard: Tout-Monde. Paris 1993; tr. it.: Tutto-mondo. Roma 2009.

Glissant, Èdouard: La cohée du Lamentin - Poetique V. Paris 2005, tr. it.: Il pensiero del tremore. Milano 2008.

Glissant, Èdouard: Poetica della Relazione. Poetica III. Macerata 2007.

Glissant, Èdouard: Traité du Tout-Monde. Poétique IV. Paris 1997.

Jacob, Christian: L'empire des cartes: Approche théorique de la cartographie à travers l'histoire. Paris 1992.

Neve, Mario: Il disegno dell'Europa. Costruzioni cartografiche dell'identità europea. Milano / Udine 2016.

Neve, Mario: “Geoestetica della scoperta”, in: Geotema (27) 2008, pp. 165-176.

Neve, Mario: “'Milieu', luogo e spazio. L'eredità geoestetica di Simondon e MerleauPonty", in: Chiasmi International, nuova serie (7) 2005, pp. 153-170.

Neve, Mario: "Sensorium communis geographiae. Some introductory steps to a geoaesthetics", in: Ocula (5) 2004, pp. 1-21.

Neve, Mario: Itinerari nella geografia contemporanea. Roma 2004.

Prato, Gian Luigi: "Il pluralismo geografico del cosmo abitato: i settanta popoli della terra (Gen 10)", in: id.: Gli inizi e la storia. Le origini della civiltà nei testi biblici. Roma 2013, pp. 135-160.

Prato, Gian Luigi: Identità e memoria nell'Israele antico. Storiografia e confronto culturale negli scritti biblici e giudaici. Brescia 2010.

Prato, Gian Luigi: "La ‘torre di Babele' e la comunicazione interrotta. Pluralità di lingue o ambiguità del linguaggio?", in: Ciola, Nicola / Pulcinelli, Giuseppe (a cura di): Nuovo Testamento: teologie in dialogo culturale. Scritti in onore di Romano Penna nel suo $70^{\circ}$ compleanno. Bologna 2008, pp. 53-62.

Prato, Gian Luigi: "Dalla geografia neutrale alla mappa ideologica centralizzata. La 'tavola dei popoli' (Gen 10) da testo delle origini a immagine normativa", in: Cagni, Luigi (a cura di): Biblica et Semitica. Studi in memoria di Francesco Vattioni. Napoli 1999, pp. 511-546.

Prontera, Francesco: Geografia e storia nella Grecia antica. Firenze 2011. 
Prontera, Francesco: "Geografia e corografia: note sul lessico della cartografia antica", in: Pallas (72) 2006, pp. 75-82.

Prontera, Francesco (a cura di): Geografia e geografi nel mondo antico. Guida storica e critica. Roma / Bari 1983.

Quirós, Kantuta / Imhoff, Aliocha (ed.): Géoesthétique. Paris 2014.

Sacchi, Paolo: Apocrifi dell'Antico Testamento. Vol. I. Torino 1981, pp. 258-270.

Said, Edward Wadie: Orientalism. New York 1978; tr. it.: Orientalismo. L'immagine europea dell'Oriente. Milano 2002.

Van Paassen, Christiaan: "L'eredità della geografia greca classica: Tolomeo e Strabone”, in: Prontera, Francesco (a cura di): Geografia e geografi nel mondo antico. Guida storica e critica. Roma / Bari 1983, pp. 227-273.

\section{Immagini}

Fig. 1: Imago mundi della tradizione cristiana medievale, disponibile su: https://de.wiki pedia.org/wiki/Radkarte\#/media/File:Radkarte_MKL1888_edited_(T_and_O_style, east-up).png [24.10.2016].

Fig. 2: Mappa mundi di Isodorus' Etymologiae, disponibile su: https:/it.wikipedia.org/ wiki/Etymologiae\#/media/File:Etymologiae_Guntherus_Ziner_1472.jpg [24.10.2016].

Fig. 3: Mappa mundi di Isodorus' Etymologiae, disponibile su: https://4.bp.blogspot. com/-Q-eak_qz18Y/VGJB8mVwV6I/AAAAAAAAxrI/afX0YrGldo4/s1600/mappa mondo\%2BT-O.jpg [24.10.2016].

Fig. 4: EurO\&T (2011), disponibile su: http://annemoma.com/exhibitions/4812/archi scriptons/works/205952/euro-t/ [24.10.2016]. 